\title{
Location-Based Adaptation for DASH in Vehicular Environment
}

\author{
Ayub Bokani \\ School of Computer Science and Engineering \\ The University of New South Wales, Sydney, NSW, Australia \\ National ICT Australia (NICTA) \\ abokani@cse.unsw.edu.au
}

\begin{abstract}
In dynamic adaptive streaming over HTTP (DASH), a video file is encoded at different bitrates and segmented into small chunks. Each time, the most appropriate bitrate will be selected for a video chunk based on the network condition observed by streaming client. This may lead to video freezing or not efficiently using the network capacity due to unexpected bandwidth changes especially in fast moving environment. In this research we take advantage of geographical knowledge of bandwidth to predict the network behavior in next moments. Using simulation driven by real-world $3 \mathrm{G}$ bandwidth and vehicular mobility traces we achieved higher QoS when geographical throughput history was considered.
\end{abstract}

\section{Keywords}

Adaptive Video Streaming; Bandwidth Map

\section{INTRODUCTION}

HTTP-based delivery has became the first choice of content providers to deliver different types of contents such as video to their clients within recent years. Different streaming platforms including Apple's HTTP Live Streaming (HLS) [2], Microsoft's Smooth Streaming [13] and Adobe's HTTP Dynamic Streaming (HDS) [1] were developed with this purpose. In these platforms, a given video file is encoded in multiple bitrates (quality levels). Then, it will be segmented into a sequence of small video chunks of typically 2-10 second duration. These video chunks will be stored on the media server separately.

At the beginning of streaming session, a manifest file containing ' $u r l$ 's of all video chunks in different qualities is sent to the streaming client. Using the standard HTTP GET command, client fetches and plays video chunks with respecting to their sequential order. Each chunk is required

\footnotetext{
* This research is supervised by Prof. Mahbub Hassan and A/Prof. Salil Kanhere.
}

Permission to make digital or hard copies of all or part of this work for personal or classroom use is granted without fee provided that copies are not made or distributed for profit or commercial advantage and that copies bear this notice and the full citation on the first page. Copyrights for components of this work owned by others than ACM must be honored. Abstracting with credit is permitted. To copy otherwise, or republish, to post on servers or to redistribute to lists, requires prior specific permission and/or a fee. Request permissions from permissions@ acm.org.

CoNEXT Student Workshop'14, December 2, 2014, Sydney, Australia.

Copyright 2014 ACM 978-1-4503-3282-8/14/12 ...\$15.00.

http://dx.doi.org/10.1145/2680821.2680836. to be buffered in the client device before its playback deadline to avoid 'freezing' effects. Based on available bandwidth throughput observed by client, the downloading times of next video chunk in available bitrates will be approximated. The highest possible quality level that ensures a smooth video playback at the receiver will be selected.

To facilitate wide-spread deployment of this technology, a standard, called dynamic adaptive streaming over HTTP (DASH) [9], is introduced by the world wide web consortium (W3C). The DASH standard only specifies how the video chunks should be stored and what metadata about the chunks should be provided to a client. The actual streaming strategy, i.e., the client intelligence for selecting the right quality for each chunk in order to produce a high quality of experience (QoE) for the viewer is left to the developers.

In this paper, we discuss how the streaming client optimizes streaming quality by getting advantage of bandwidth statistics that can be mapped to the urban road networks.

\section{BANDWIDTH MAP}

Despite of considerable improvements in wireless networks' capacity, the instability and inconsistency of bandwidth still are in question. Interference, fading, scheduling issues or other effective parameters make bandwidth to fluctuate over time and location and behave like a random variable not a deterministic entity. Therefore, sustaining the QoS in mobile environment is a challenging problem.

With lower degree, the bandwidth is known to fluctuate even in wired networks [8]. This, raised a significant research interest on network behavior prediction for different applications. However, some domains such as multimedia streaming require a specified minimum bandwidth to provide a real-time service for the end users. Due to significant bandwidth fluctuation over different geographical locations, a fast moving client who is watching a video stream and passes different locations may suffer from video freezing or quality fluctuations. The video freezing is occurred when the available bandwidth is less than what is required for a smooth playback and the quality fluctuation is the consequence of bitrate adaptations.

Without bandwidth distribution knowledge, the adaptation is done based on network observations during the streaming session which gives a global knowledge of network behavior. Yao et al. [11] and Deshpande et al. [5] have collected bandwidth traces from $3 \mathrm{G}$ networks while driving a car and using an entropy-based method have shown that road-segment-based statistics contain more information about bandwidth compared to global statistics. Figure 1a demon- 


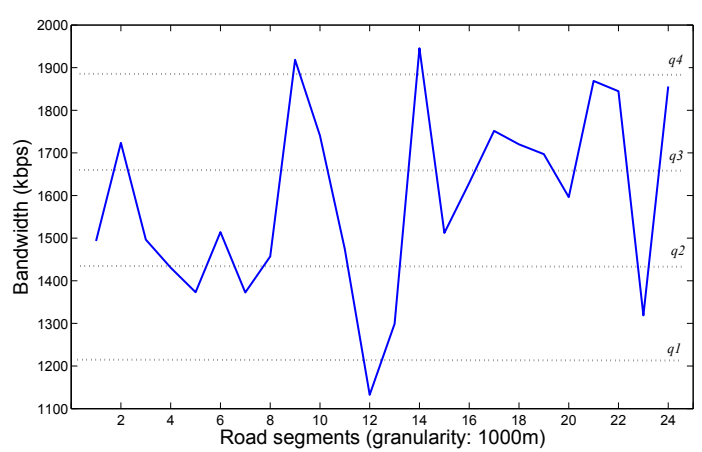

(a)

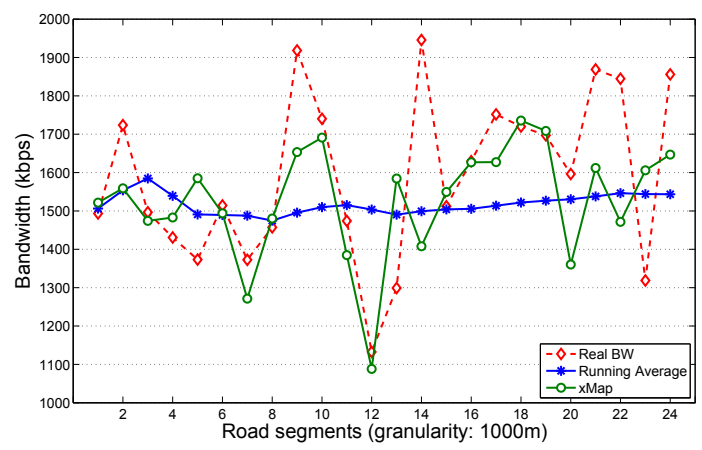

(b)

Figure 1: Bandwidth fluctuation in different road segments (a), Comparison between bandwidth history and observed bandwidth (b)

strates the bandwidth variation in different road segments. As it's shown, from segment 1 to 10 of the road, relying on observed network throughput, the adaptation program tries to remain on quality 3 and 2 . In segment 12 where the bandwidth drops below the required amount for these qualities, video freezing event is expected. Having the historical information of bandwidth samples of segment 12, makes it possible to predict the drop before reaching that point. As it's illustrated in Figure 1b, the average of historical bandwidth samples in most of the road segments is much closer to the real available bandwidth compare to the running average of observed throughput. In this research, we use the bandwidth dataset provided by Yao et al. [11] containing 71 traces on $3 \mathrm{G}$ networks in a $22 \mathrm{~km}$ route from University of New South Wales to the Macquarie University in Sydney.

\section{CLIENT INTELLIGENCE}

Although with the historical information of bandwidth we can predict the network behavior in different locations [12], in such stochastic environment a considerable prediction error is expected. Therefore, to improve the QoS of video streaming, we consider Markov Decision Process (MDP) as a well-known optimization framework in such stochastic scenarios. In the literature, streaming rate adaptation has been modelled with MDP in various forms and complexities. In this study, we mainly follow the formulation of $[6,10]$ with some modifications [4] to consider bandwidth maps and deal with the MDP computation overhead.

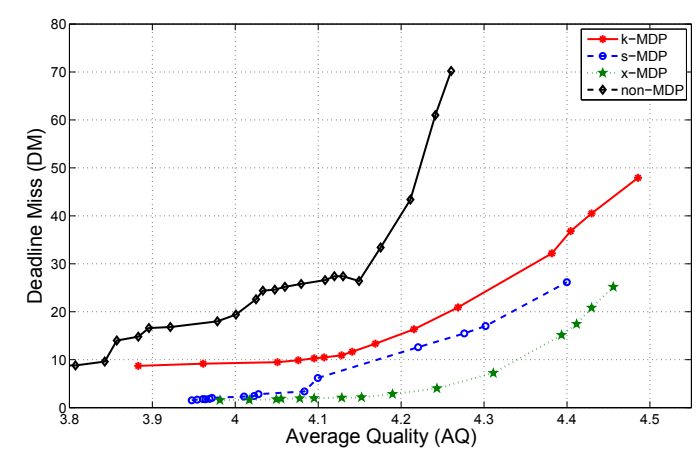

Figure 2: Video streaming performance: Comparing nonMDP, online MDP $(k$-MDP) and offline MDP $(s-\mathrm{MDP}$ and $x$-MDP) algorithms - video clip: Big Buck Bunny [3], length: 09:56 repeated within $22 \mathrm{~km}$ simulated trips

We consider both on-line and offline MDP optimizations. For online, we propose a $k$-chunk update approach ( $k$-MDP) which recomputes the optimal strategy after downloading every $k$ chunks. The online approach uses collected bandwidth statistics during the streaming session without referring to any offline statistics. For offline, we propose two different approaches, single MDP strategy ( $s$-MDP) and $x$ meter multiple MDP strategy $(x$-MDP). $s$-MDP uses the global statistics of a given region to compute an optimal MDP strategy which is used throughout the video session in any given trip in that region, while $x$-MDP recomputes the optimal strategy for every $x$ meters of the route using offline statistics for the last $x$-meter of the road.

We have evaluated the performance of a non-MDP algorithm (i.e., Rate Adaptation [7]) and proposed MDP approaches using simulation driven by real-world $3 \mathrm{G}$ bandwidth and vehicular mobility traces. As it's shown in Figure 2, all MDP algorithms outperform the non-MDP by missing less playback deadlines for similar quality level. We also find that $k$-MDP is capable of reducing online computation overhead significantly without any noticeable degradation in QoE. Interestingly, although offline approaches have zero online computation overhead, they both outperform the online approach. The best performance is achieved with $x$-MDP.

\section{CONCLUSIONS}

In this paper we have evaluated the advantage of using geographical bandwidth history for adaptive video streaming. With this method, the network behavior in next moments can be predicted much more precisely compare to other conventional methods. We have also considered three approaches to reduce the computation overhead of DASH when MDP is used as the underlying optimization framework. Conceptually, the on-line MDP overhead could be completely eliminated with off-line optimization using only past observations of mobile bandwidth for a given region or road segment. Our simulation experiments have revealed that such pure offline MDP optimizations outperform the on-line optimization in terms of improved trade off for picture quality and deadline miss. The best performance is achieved when the MDP strategy is optimized using the bandwidth CDF of each specific road segment, which allows the system to realign with any statistical differences between different locations. 


\section{REFERENCES}

[1] Adobe. HTTP Dynamic Streaming on the Adobe Flash Platform. [Online accessed 04-March-2013], URL: http://www.adobe.com/au/products/hdsdynamic-streaming.html.

[2] Apple. HTTP Live Streaming Overview. [Online accessed 01-October-2014], URL: http://goo.gl/CElrwV.

[3] BlenderFoundation. Big Buck Bunny. [Online accessed 04-March-2013], URL: http://www.bigbuckbunny.org.

[4] A. Bokani, M. Hassan, and S. Kanhere. HTTP-based Adaptive Streaming for Mobile Clients using Markov Decision Process. In Packet Video Workshop (PV), San Jose, USA. 12 December 2013.

[5] P. Deshpande, X. Hou, and S. R. Das. Performance Comparison of $3 \mathrm{G}$ and Metro-Scale WiFi for Vehicular Network Access. In Proceedings of the 10th ACM conference on Internet measurement, Melbourne, Australia. 1âĂŞ3 November 2010.

[6] D. Jarnikov and T. Özçelebi. Client Intelligence for Adaptive Streaming Solutions. Signal Processing: Image Communication, 26(7):378-389, 2011.

[7] C. Liu, I. Bouazizi, and M. Gabbouj. Rate Adaptation for Adaptive HTTP Streaming. In Proceedings of the second annual ACM conference on Multimedia systems (MMSys' 11), New York, USA. 23 February 2011.
[8] Y. Qiao, J. Skicewicz, and P. Dinda. An Empirical Study of the Multiscale Predictability of Network Traffic. In Proceedings of the 13th IEEE International Symposium on High Performance Distributed Computing (HPDC-13), Honolulu, Hawaii, USA. June 2004.

[9] T. Stockhammer. Dynamic Adaptive Streaming Over HTTP: Standards and Design Principles. In Proceedings of the second annual ACM conference on Multimedia systems (MMSys), San Jose, USA. 23-25 February 2011.

[10] C. C. Wüst and W. F. Verhaegh. Quality Control for Scalable Media Processing Applications. Journal of Scheduling, 7(2):105-117, 2004.

[11] J. Yao, S. S. Kanhere, and M. Hassan. An Empirical Study of Bandwidth Predictability in Mobile Computing. In Proceedings of the third ACM international workshop on Wireless network testbeds, experimental evaluation and characterization (MobiCom-WiNTECH), San Francisco, USA. 14-19 September 2008.

[12] J. Yao, S. S. Kanhere, and M. Hassan. Improving QoS in High-Speed Mobility Using Bandwidth Maps. IEEE Transactions on Mobile Computing, 11(4):603-617, 2012.

[13] A. Zambelli. IIS smooth streaming technical overview. Microsoft Corporation, 3, 2009. 